\title{
Do galactic globular clusters contribute to microlensing events?
}

\author{
de Luca, F ; Jetzer, P
}

\begin{abstract}
In this paper, we perform an analysis of the large set of microlensing events detected so far toward the galactic center, with the purpose of investigating whether some of the dark lenses are located in galactic globular clusters. We found that in four cases some events might have occurred due to lenses located in the globular clusters themselves. We also give a rough estimate for the average lens mass of the subset of events being highly aligned with galactic globular-cluster centers and find that, under reasonable assumptions, the deflectors could possibly be either brown dwarfs, M-stars, or stellar remnants.
\end{abstract}

DOI: https://doi.org/10.1142/S0218271808013868

Posted at the Zurich Open Repository and Archive, University of Zurich

ZORA URL: https://doi.org/10.5167/uzh-17012

Journal Article

Accepted Version

Originally published at:

de Luca, F; Jetzer, P (2008). Do galactic globular clusters contribute to microlensing events? International Journal of Modern Physics. D, 17(12):2305-2314.

DOI: https://doi.org/10.1142/S0218271808013868 


\title{
Galactic globular clusters contribution to microlensing events?
}

\author{
Fabiana De Luca and Philippe Jetzer \\ Institute for Theoretical Physics, University of Zürich, Winterthurerstrasse 190, \\ CH-8057 Zürich, Switzerland
}

\begin{abstract}
In this note we perform an analysis of the large set of microlensing events detected so far toward the Galactic center with the purpose of investigating whether some of the dark lenses are located in Galactic globular clusters. We find that in four cases some events might indeed be due to lenses located in the globular clusters themselves. We also give a rough estimate for the average lens mass of the subset of events being highly aligned with Galactic globular cluster centers and find that, under reasonable assumptions, the deflectors could most probably be either brown dwarfs, M-stars or stellar remnants.
\end{abstract}

Key words: Gravitational lensing - Galaxy: globular cluster

\section{Introduction}

Microlensing event analyses have been originally proposed as a tool to detect dark matter in form of MACHOs (MAssive Compact Halo Objects) (Paczynski 1986). A remarkable number of collaborations has put considerable effort in the search for such events toward various targets and a large amount of detections has been collected. Since the lens mass and velocity distributions are tightly related with the spatial and time distributions of the events, the latter can be a useful tool to understand the structure, dynamics and the initial mass function in the low mass range of our own galaxy, as shown for instance in Wood \& Mao (2005) and Calchi Novati et al. (2007) for the Milky Way.

Globular cluster could contain a sizeable amount of dark matter in form of brown dwarfs or low mass stars. This is still an open issue and a possible way to test this is to use microlensing observations as suggested by Paczynski 
(1994). The idea is to monitor globular clusters in front of rich background of either the SMC, in the case of 47 Tuc, or the galactic bulge. In this case, when the lens belongs to the cluster population, its distance and velocity are roughly known. This way it is possible to get a more accurate estimate for the lens mass. Such a study has already been performed by Jetzer et al. (1998) for the globular clusters in the direction of the galactic bulge region. Indeed, some events were found which might be associated with lenses in globular clusters (Jetzer et al. (1998); Sahu et al. (2001)). However, given the few events at disposal it has not been possible to draw firm conclusions. In the meantime the number of microlensing events detected toward the galactic bulge region has considerably increased and it is thus reasonable to repeat that analysis. We do not consider here the possibility that the source lies as well in the globular cluster, as its probability is much smaller, nonetheless such suggestions have been also studied (see also Gyuk \& Holder (1997); Rhoads \& Malhotra (1998); Cardone \& Cantiello (2003)).

In this note we analyse the possible MACHO content in a large set of Galactic Globular Clusters (hereafter GGCs) some of which are highly aligned with a non negligible number of microlensing events detected toward the Galactic Center (hereafter GC).

The basic consideration is that, since the observed event duration (Einstein time, $\left.t_{E}\right)$ is a function of the lens mass $t_{E} \sim m^{1 / 2}$, an estimation of the latter can be drawn through reasonable assumptions on the spatial and velocity distributions of the lens and source populations.

Our aim, following the previous analysis in Jetzer et al. (1998), is to get more stringent conclusions by enlarging the analysed data set that now includes 4697 microlensing events detected in the last years by the MACHO (Thomas et al. 2005), EROS (Hamadache et al. 2006), OGLE (Sumi et al. (2006), http : //www.astrouw.edu.pl/ ogle/), and MOA (http://www.phys.canterbury.ac.nz/moa/) collaborations in direction of the GC.

This note is structured as follows. An overview of the adopted models for the Galactic luminous components and GGCs is given in Sect. 2, the results are discussed in Sect. 3 and Sect. 4 is devoted to the conclusions.

\section{Models}

In our analysis we focus on the configuration in which the lens is hosted in a GGC and the source is located either in the Galactic disc or bulge (Taillet et al. (1995), Taillet et al. (1996)). The possibility that the source belongs to a GGC is neglected because GGC centers are very crowded regions, with 
at most $10^{5}$ stars, often located toward the GC, this making very unlikely the detection of microlensing of a GGC star, since very high resolutions and long observation periods (due to the very small optical depth) are required to provide valueable results.

As regards the GGCs, the mass density of their luminous component is well described by a King function (King 1962)

$$
\rho(r)=\frac{\rho(0)}{z^{2}}\left[\frac{\arccos [z]}{z}-\sqrt{1-z^{2}}\right], \quad z=\sqrt{\frac{r_{c}^{2}+r^{2}}{r_{c}^{2}+r_{t}^{2}}},
$$

that corresponds to a spherically symmetric mass distribution whose surface density decreases with distance from the center and rapidly drops to zero from $r=r_{t}$ on, as supported by star counts (for more details see Binney \& Tremaine (1987); Jetzer et al. (1998)).

In eq. (1) $r_{c}$ and $r_{t}$ are the GGC core and tidal radius, respectively, whose values are as given in Harris (1996), while $\rho(0)$ has been calculated from the total GGC mass given in Mandushev et al. (1991). In particular, out of the initial 150 GGCs given in Harris (1996), we will only focus on a subset of 135 clusters for which the crossing of the two catalogs provides the complete set of necessary parameters.

Since the MACHO distribution in GGCs is not known, we assume for the dark matter the same mass density profile as given in eq. (1) but rescaled by a factor $f=\frac{M_{\text {dark }}}{M_{\text {tot }}}$ which is the fraction of the total MACHO mass on the overall GGC mass, that can even get the remarkable value of $1 / 2$ (Heggie \& Hut 1995).

For the Galactic bulge we assume a density profile, $\rho_{b}$, as found in Stanek et al. (1997), where the model that best fits the observations suggests a triaxial, boxy shaped bulge with $\rho_{b}=\rho_{0, b} \exp [-r], r=\sqrt{\left(x / x_{o}\right)^{2}+\left(y / y_{o}\right)^{2}+\left(z / z_{o}\right)^{2}}$ and major semiaxes $\left\{x_{o}, y_{o}, z_{o}\right\}=\{0.897,0.387,0.250\} p c$, the major axis being clockwise rotated on the Galactic plane of an angle $\alpha=23.8^{\circ}$ with respect to the direction Sun-GC (Calchi Novati et al. 2007).

Finally, the Galactic disc density profile is assumed to be exponentially decreasing in both the Galactic plane and vertical direction with $\rho_{d}=\rho_{0, d} \exp [-$ $\left.\frac{R-R_{\odot}}{H}\right] \operatorname{sech}^{2}\left[\frac{z}{h}\right]$, where $\{R, z\}$ are the distance from the GC on the equatorial plane and the height above it, respectively, $R_{\odot}=8 \mathrm{kpc}$ is the distance of the Sun from the GC and $\{H, h\}=\{2.75,0.250\}$ kpc. Our assumption on the Galactic disc structure closely follows the model adopted by Han \& Gould (2003) except for the fact that we neglect the contribution of a thick disc since this component should only provide minor contribution to the overall disc density (Vallenari et al. 2006). For further details on the bulge and disc density functions we refer to Calchi Novati et al. (2007). 


\section{Results}

Aiming at discriminating among events due to lenses hosted either in GGCs or in the Galactic bulge/disc, we first make a rough selection of events being aligned with a GGC. In particular, for every given GGC, we consider a sphere of radius $r_{t}$, centered at the GGC center, and we select, as a first step, only the events being included in one such contour, as shown in Fig. 1. By doing so, out of the original 4697 events, we are left with only 118.

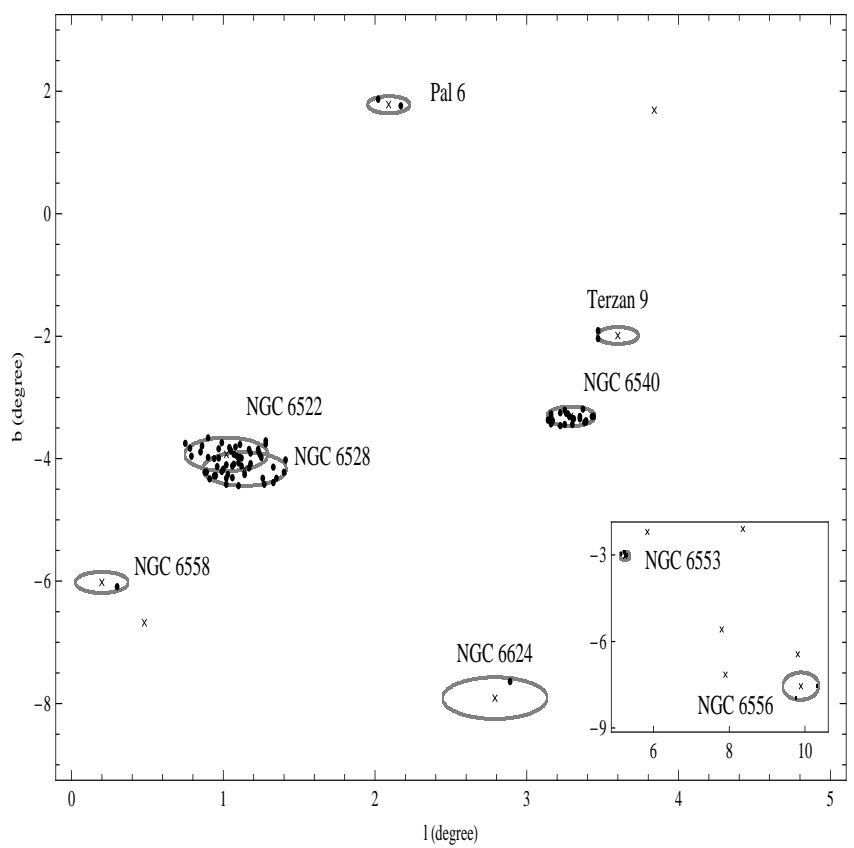

Fig. 1. The points mark the MACHO, EROS, OGLE and MOA events detected behind GGCs. The crosses denote the centers of the GGCs. Solid lines: contours on the sky plane of the sphere of radius equal to the cut radius of the GGC.

The GGCs aligned with at least one event are given in Table 1. Some properties are also reported, such as the tidal and the core radius, the distance from the Sun (Harris 1996) and the number of included events. Moreover we give the optical depth, $\tau$, (Kiraga \& Paczynski 1994; Calchi Novati et al. 2007)

$$
\tau=\frac{\frac{4 \pi G}{c^{2}}}{\int_{0}^{\infty} \rho_{s} d D_{o s}} \int_{D_{o s}}^{\infty} \rho_{s} d D_{o s} \int_{0}^{D_{o s}} \rho_{l} D_{o l}\left(1-\frac{D_{o l}}{D_{o s}}\right) d D_{o l}
$$

calculated toward the GGC centers for sources located in the Galactic bulge/disc and lenses belonging to the GGC. Here $D_{o l}\left(D_{o s}\right)$ is the observer-lens (observersource) distance as measured along the line of sight observer-source (hereafter l.o.s.). The different core radii and central densities explain the large variations of the optical depth among the GCCs. It is worth to underline that for 
Table 1

GGCs being aligned with at least one detected microlensing event. For each of them the number of aligned events $N_{t o t}$ and the corresponding average duration $\left\langle t_{E}\right\rangle$ (in days) is given. For every GGC, $r_{t}$ is the tidal radius (in pc), $r_{c}$ is the core radius (in pc), $r_{\text {sun }}$ is the distance of its center from the Sun (in kpc) and $\tau$ is the optical depth toward its center in units of $f \times 10^{-5}, f$ being the fraction of dark matter mass in the GGC (see section 2 for details).

\begin{tabular}{c|cccccc}
\hline Cluster ID & $r_{\text {sun }}$ & $r_{t}$ & $r_{c}$ & $\tau$ & $N_{\text {tot }}$ & $\left\langle t_{E}\right\rangle$ \\
\hline Pal 6 & 5.9 & 14.3 & 1.13 & 0.24 & 2 & 76.3 \\
Terzan 9 & 6.5 & 15.5 & 0.06 & 0.78 & 2 & 17.8 \\
NGC 6522 & 7.8 & 37.3 & 0.11 & 6.06 & 36 & 16.8 \\
NGC 6528 & 7.9 & 38.1 & 0.21 & 1.01 & 38 & 25.2 \\
NGC 6540 & 3.7 & 10.2 & 0.03 & 23.74 & 29 & 22.8 \\
NGC 6553 & 6.0 & 14.2 & 0.96 & 1.01 & 7 & 43.0 \\
NGC 6558 & 7.4 & 22.5 & 0.06 & 7.20 & 1 & 24.5 \\
NGC 6624 & 7.9 & 47.2 & 0.14 & 4.43 & 1 & 223.0 \\
NGC 6656 & 3.2 & 27.0 & 1.32 & 0.75 & 2 & 112.7 \\
\hline
\end{tabular}

Table 2

GGCs including at least one inner event. For each of them $N_{i n}$ is the number of events inside a projected radius $r=2 \times r_{t} / 5$ and, for this subset of aligned events, $\left.<t_{E}\right\rangle$ is the mean Einstein time (in days) and $\langle m\rangle$ is the average predicted lens mass in units of solar masses. $N_{G G C}\left(N_{B D}\right)$ is the number of events, out of $N_{i n}$, that we expect to be due to GGC (Galactic bulge/disc) lenses. $\Gamma_{\text {exp }}$ is the expected event rate in units of $f \times \mu_{o}^{-1 / 2} \times 10^{-3}$ year (see text for details) while $n_{G G C}$ is $N_{G G C}$ per unit area (in degree ${ }^{-2}$ ).

\begin{tabular}{c|ccccccc}
\hline Cluster ID & $N_{i n}$ & $\left\langle t_{E}\right\rangle$ & $\langle m\rangle$ & $N_{B D}$ & $N_{G G C}$ & $\Gamma_{\exp }$ & $n_{G G C}$ \\
\hline NGC 6522 & 8 & 13.1 & 1.63 & $4.1 \pm 2.0$ & 3.9 & 0.66 & 51.4 \\
NGC 6528 & 7 & 13.0 & 2.98 & $4.9 \pm 2.2$ & 2.1 & 0.09 & 27.5 \\
NGC 6540 & 7 & 17.2 & 0.06 & $4.2 \pm 2.0$ & 2.8 & 1.56 & 112.3 \\
NGC 6553 & 4 & 35.7 & 0.62 & $0.6 \pm 0.8$ & 3.4 & 0.08 & 185.4 \\
\hline
\end{tabular}

both lenses and sources belonging to a GGC, the optical depth is 2-3 orders of magnitude smaller than the reported $\tau$, while the introduction of a thick disc (see Calchi Novati et al. (2007) for a modelisation) only implies variations of the order of $5 \%$ or less.

Due to the GGC structure, we expect the predicted number of events to be the largest toward their centers and to decrease as we move toward their borders. Since the alignment between an event and a projected cluster contour does not 
assure that the deflector belongs to the GGC, this alignment possibly being accidental, we make a further, rough selection and consider only the events being included in the projected contour of a sphere centered at a GGC center and of radius $r_{i}=2 \times r_{t} / 5$ (this including on average $90 \%$ of the total cluster mass). We then distinguish between inner and outer events, the former being inside $r_{i}$ and the latter being included in the circular ring of internal radius $r_{i}$ and outer radius $r_{t}$. By doing so, we assume all the outer events to be due to Galactic bulge/disc deflectors (this possibly underestimating the events due to GGC lenses), whereas we leave open the possibility that among the inner events some could still be attributed to bulge/disc deflectors. Object of our analysis is the subset of 28 inner events that we are left with, among which $7(17 / 4)$ have been detected by the MACHO (OGLE/MOA) collaboration. Notice that, as shown in Fig. 1, the projections of NGC 6522 and NGC 6528 superimpose and have some aligned events in common. The latter are nevertheless far from the centers of both GGCs and are not included in our analysis.

An estimation of the predicted number of events, $N_{G G C}$, due to MACHOs in a given GGC, can be roughly made as follows. Assuming that all the outer events are due to Galactic bulge/disc lenses, we calculate how many such events, $N_{B D}$, are expected in the inner region of a GGC contour assuming that the number of events is proportional to the covered area and that the background source distribution is uniform inside every GGC contour. Thus we assume that the microlensing rate for Galactic bulge/disc events is constant over the entire small area within the tidal radius of the considered globular cluster. By doing so, $N_{B D}$ is simply proportional to the monitored area. Clearly, also with these assumptions, which are reasonable, given the very small area considered, one expects fluctuations in the number of events in a given area. We assume the fluctuations to follow Poisson statistics, in which case they are given by $\sim \sqrt{N_{B D}}$. By doing so, for every GGC considered, $N_{G G C}$ turns out to be around 2-4 per cluster (see Table 2) and in two cases this number is larger than the estimated fluctuation of $N_{B D}$. Given these numbers we cannot claim for any clear evidence of lenses hosted in GGCs. Nonetheless, it is remarkable that for the 4 cases considered the value of $N_{G G C}$ is positive and most probably underestimated, since the assumption that all the events lying in the outer ring are due to bulge/disc deflectors possibly overestimates $N_{B D}$.

The fact that some events can be attributed to GGC MACHOs can be further corroborated by the following consideration. For a given GGC, we consider five circles of radius $r_{i}=i \times r_{t} / 5(\mathrm{i}=1, . ., 5)$ and centered at the GGC center. In every circle we compute the event density, $n_{o b s, i} \equiv N_{o b s, i} / A_{i}, N_{o b s, i}$ being the number of detected events inside the $i$-th circle and $A_{i}$ being the area of the $i$-th circle. For every GGC considered, the bar heights have been normalized to $\sum_{i=1}^{5} n_{o b s, i}=1$. 


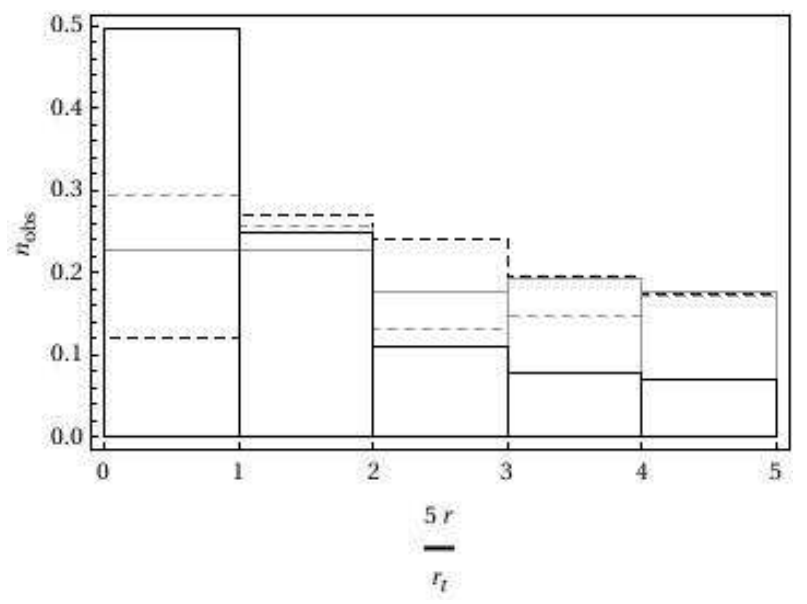

Fig. 2. Barcharts of the number of detected events per unit area as a function of distance from the center of the GGCs of Table 2. For every GGC of Table 2, we have divided its contour of radius $r=r_{t}$ in five circles centered at the GGC center and with radius $r_{i}=i \times r_{t} / 5,(\mathrm{i}=1, . ., 5)$. In each of these circles we have calculated the number of detected events per unit area and have normalized the resulting barchart to unity (see text for more details). Black (solid,dashed) and gray (solid,dashed) lines show the barchart for NGC 6553, NGC 6522, NGC 6528 and NGC 6540, respectively.

Fig. 2 shows the barcharts obtained for all the GGCs of Table 2. In particular, for NGC 6553 (solid, black line) there is a clear excess of observed events toward its center. The same trend is found for NGC 6528 and NGC 6540 even though with less steep slopes, while NGC 6522 shows an inverse trend only in its innermost circle. Notice, anyways, that for all the considered GGC between $40 \%$ to $80 \%$ of the events are located within $r=2 \times r_{t} / 5$ from the GGC center.

Assuming that the deflector is a GGC MACHO, we can estimate its mass through the relation $R_{E} / t_{E}=v_{r}$, where $v_{r}$ is the lens-source relative velocity orthogonal to the l.o.s., $t_{E}$ is the event Einstein time and $R_{E}=\sqrt{\frac{4 G}{c^{2}} m D_{o l}\left(1-\frac{D_{o l}}{D_{o s}}\right)}$ is the Einstein radius, $m$ being the deflector mass. As reported in Harris (1996), the mean GGC tidal radius is of the order of tens of pc, this making the GGC extension relatively small compared to the average $D_{o l}$ and $D_{o s}$ considered (of the order of kpc), since we are assuming Galactic bulge/disc sources and the GGCs are kpcs away from the Sun. For this reason, we make the simple assumption that in a given GGC the MACHOs are all at the same distance from the Sun $\left(r_{\text {sun }}\right.$, as given in Table 1). Furthermore, we assume all the sources to be on the Galactic $\{y, z\}$-plane (Jetzer et al. 1998). As regards to $v_{r}$, we take advantage of recent results. In particular, except NGC 6540, all the GGCs listed in Table 2 have been object of deep investigation and their proper motion has been described with considerable accuracy (Terndrup et al. 1998; Zoccali et al. 2001, 2003; Feltzing \& Johnson 2002; Dinescu et al. 2003). In particular, in the reference frame $(U, V, W)$ where $U$ is positive outward from the GC toward the Sun, $V$ is positive toward the Galactic rotation 
and $W$ is positive toward the Galactic Pole, we have $(27,57,-227) \mathrm{km} / \mathrm{s}$ for NGC 6522, (-197, -26,4) km/s for NGC 6528 and $(9,225,14) \mathrm{km} / \mathrm{s}$ for NGC 6553. Moreover, due to the lack of information, we assume for NGC 6540 the reference value $v_{r}=100 \mathrm{~km} / \mathrm{s}$ and remind that for any other value, $\tilde{v}_{r}$, of the orthogonal relative velocity, the corresponding lens mass, $\tilde{m}$, is simply rescaled as $\tilde{m}=\left(\frac{\tilde{v}_{r}}{v_{r}}\right)^{2} m$. Table 2 shows, for the whole subset of inner events, the predicted deflector mass in units of solar masses, $\langle m\rangle$, obtained with these assumptions. The resulting average lens mass gets values in the range $\left\{10^{-2}, 10\right\}$, suggesting that the involved deflectors are possibly either brown dwarfs, M-stars or stellar remnants. Moreover, Jupiter-like deflectors are not definitively excluded, since, already a small increase on $D_{o s}$ can substantially reduce the predicted lens mass ${ }^{1}$.

The bar chart of the values of the expected lens mass for the inner events is shown in Fig. 3, which highlights a crowding at $\sim 1 M_{\odot}$. The differences between the predicted $<m>$ that are given here and the ones shown in Jetzer et al. (1998) are due to the different Einstein time distributions, to the more appropriate GGCs proper motions adopted here and to the fact that they assume $D_{o s}=8.5 \mathrm{kpc}$ while we have $D_{o s} \sim 8.1 \mathrm{kpc}$, with a very small dispersion among the targets.

The average expected lens mass has been drawn from the set of inner events, some of which being possibly not due to GGC MACHOs. This source of contamination should be removed before one makes any prediction, but since we are not able to do such a distinction, the average values on the overall inner sample can be taken as a first crude approximation.

Also given in Table 2 is the number of expected events toward the GGC centers, $\Gamma_{\text {exp }}$, as calculated through formula (36) of Jetzer et al. (1998), where it is assumed that all the lenses have the same mass, $\mu_{o}$, in units of solar masses and that their distribution is very narrow with respect to that of the source population. $\Gamma_{\text {exp }}$ is given in units of $f \times \mu_{o}^{-1 / 2} \times 10^{-3} /$ year, $f$ being the fraction of dark matter (in form of brown dwarfs, dim stars or stellar remnants) in the cluster. For a typical value of $10^{2}-10^{3}$ monitored source stars behind a GGC (this number depending also on the GGC extension) and

1 Following our analysis we find for both NGC 6522 and NGC 6528 an inner event whose predicted mass $m$ would exceed $10 M_{\odot}$. These large values are essentially due to the term $\left[D_{o l}\left(1-D_{o l} / D_{o s}\right)\right]^{-2}$ which takes into account the lens geometry, that, with our choice on $D_{o s}(\sim 8.1 \mathrm{kpc})$ for both NGC 6522 and NGC 6528, becomes very small, since $D_{o l}$ is almost equal to $D_{o s}$. However, this last assumption might not hold as $D_{o s}$ could be, for instance, larger, thus reducing the mass value substantially. Indeed, already a slight variation by $(\sim 2 \%)$ of $D_{o s}$ can reduce the predicted lens mass by a factor 10. Clearly, another possibility is that these two events are not due to lenses located in the GGC and thus our mass estimate does not apply. Given these uncertainties we have not reported these two events in Fig. 3. 
an observation period of $\sim 5$ to 10 years, we expect at most between half an event and a couple of events toward each GGC depending also on the value of $f$, in reasonable agreement with the results of Table 2. Notice that $\Gamma_{\text {exp }}$ for NGC 6540 is almost 15 times larger than the one predicted for NGC 6528. This is due to the larger central density of NGC 6540, which is a compact and massive GGC. On the other hand, since its extension is smaller, the number of aligned sources is quite small and thus the event rate toward its center gets reduced.

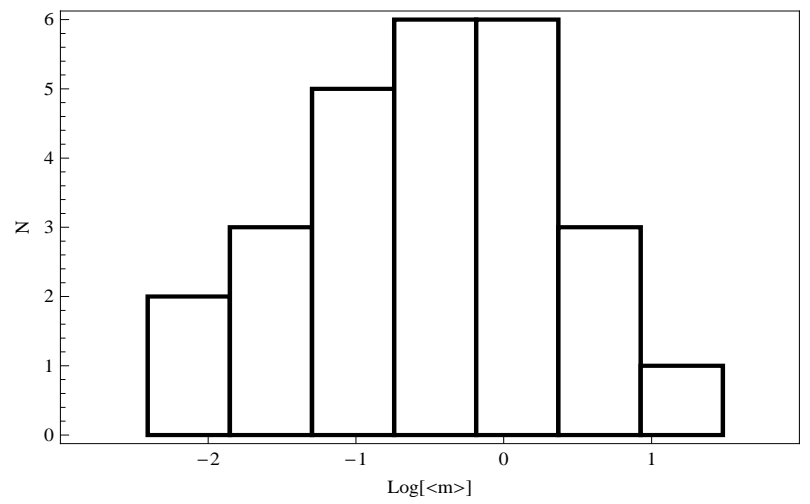

Fig. 3. Bar chart of the calculated lens mass, in units of solar masses, for the inner events.

\section{Conclusions}

We have analysed a large set of microlensing events detected toward the GC and have investigated the issue of whether and in which amount some of the observed events can be attributed to MACHOs being hosted in GGCs. We have first selected all the events being aligned with a GGC and have taken into account the fact that still many of them indeed are lenses located in the Galactic bulge/disc. We have estimated the excess of events due to MACHOs in the GGC by dividing the area of each GGC in two regions, an outer and an inner one, and assuming that all the outer events are due to lenses not being hosted in the GGC and extrapolated the number of expected bulge/disc events in the inner region. We found that in a small region around the centers of four of the 135 overall considered GGCs some of the observed events could be attributed to lenses hosted by the GGC. Although all of the four cases, where there are a substantial amount of events such as to be able to carry out our analysis, show an excess of events, which could indicate the presence of lenses in the globular clusters, we still cannot draw firm conclusions on this issue.

Since the analysis made by Jetzer et al. (1998) the number of events at disposal substantially increased, but not yet to a level such that this question can be answered in a definitive way. Clearly, the expected number of events, and 
thus the rate, is certainly quite small so that more observations are needed. A possible strategy would be to survey systematically during many years the line of sight comprising the four globular clusters which we analysed. Since we are not able to distinguish between the events due to the lenses in globular cluster from the bulge/disc ones, for the estimate of the mass we just considered the mean time duration of all events located in the inner region. In this way we found a mass range suggesting that lenses in the GGCs could possibly be brown dwarfs, M-stars or stellar remnants even though the uncertainties, especially on $D_{o s}$, that affect our lens mass predictions could also shrink the predicted mass interval and shift it toward the Jupiter-like objects domain. In spite of all the above mentioned limitations, we believe that our results, although not conclusive, suggest that some events might indeed be due to lenses located in globular clusters. A dedicated survey over many years of the above considered globular clusters could possibly resolve this issue.

F.D.L. acknowledges the Forschungskredit of the University of Zurich for financial support. The authors thank Mauro Sereno and Gaetano Scarpetta for helpful suggestions and discussions.

\section{References}

Binney, J. \& Tremaine, S. 1987, Galactic dynamics (Princeton, NJ, Princeton University Press, 1987, p. 747)

Calchi Novati, S., De Luca, F., Jetzer, P., Mancini, L. \& Scarpetta, G. 2007, to apper in A\&A, astro-ph 0711.3758

Cardone, V. \& Cantiello, M. 2003, A\&A, 405, 125

Dinescu, D. I. and Girard, T. M. and van Altena, W. F. \& López, C. E. 2003, AJ, 125,1373

Feltzing, S. \& Johnson, R. A. 2002, A\&A, 385, 67

Gyuk, G.. \& Holder, G.P. 1997, MNRAS, 297, L44

Hamadache, C., Le Guillou, L., Tisserand, P., et al. 2006, A\&A, 454, 185

Han, C. \& Gould, A. 2003, ApJ, 592, 172

Harris, W. E. 1996, AJ, 112, 1487

Heggie, D. C. \& Hut, P. 1996, IUAS 174, 303H

Jetzer, P., Strässle, M. \& Wandeler, U. 1998, A\&A, 336, 411

King, I. 1962, AJ, 67, 471

Kiraga, M. \& Paczynski, B. 1994, ApJL, 430, L101

Malhotra, S. \& Rhoads, J.E. 1998, ApJ, 495, L55

Mandushev, G., Staneva, A., \& Spasova, N. 1991, A\&A, 252, 94

Paczynski, B. 1986, ApJ, 304, 1

Paczynski, B. 1994, Acta Astronomica, 44, 235

Popowski, P., Griest, K., Thomas, C. L., et al. 2005, ApJ, 631, 879

Sahu, K.C., Casertano, L., Livio, M., et al. 2001, Nature, 411, 1022 
Stanek, K. Z., Udalski, A., Szymanski, M., et al. 1997, ApJ, 477, 163

Sumi, T., Woźniak, P. R., Udalski, A., et al. 2006, ApJ, 636, 240

Taillet, R., Longaretti, P. Y. \& Salati, P. 1995, Astroparticle Physics 4, 87

Taillet, R., Salati, P. \& Longaretti, P. Y. 1996, ApJ, 461, 104

Terndrup, D. M. and Popowski, P. and Gould, A. and Rich, R. M. \& Sadler, E. M. 1998, AJ, 115, 1476

Thomas, C. L., Griest, K., Popowski, P., et al. 2005, ApJ, 631, 906

Vallenari, A., Pasetto, S., Bertelli, G., et al. 2006, A\&A, 451, 125

Wood, A. \& Mao, S. 2005, MNRAS, 362, 945

Zoccali, M. and Renzini, A. and Ortolani, S. and Bica, E. \& Barbuy, B. 2001, AJ, 121, 2638

Zoccali, M. and Renzini, A. and Ortolani, S. and Bica, E. \& Barbuy, B. 2003, AJ, 125, 994 